\title{
EXAMPLES PERTAINING TO GEVREY HYPOELLIPTICITY
}

\author{
Michael Christ
}

\section{Introduction}

The purpose of this note is to introduce certain examples which shed light on a conjecture concerning hypoellipticity in Gevrey classes for partial differential operators with multiple characteristics.

For $s \geq 1$ and any open set $U$, let $G^{s}(U)$ denote the class of all $C^{\infty}$ functions $f$ defined in $U$, such that for each compact subset $K \subset U$ there exists $C<\infty$ such that for all $x \in K$ and all multi-indices $\alpha$,

$$
\left|\partial^{\alpha} f(x)\right| \leq C^{1+|\alpha|}|\alpha|^{s|\alpha|} .
$$

A linear partial differential operator $L$ is said be $G^{s}$ hypoelliptic in $U$ if for any open subset $U^{\prime} \subset U$ and any $u \in \mathcal{D}^{\prime}\left(U^{\prime}\right)$ such that $L u \in G^{s}\left(U^{\prime}\right)$, necessarily $u \in G^{s}\left(U^{\prime}\right)$. An operator $L$ is said to be microlocally $G^{s}$ hypoelliptic in a conic open set $\Gamma \subset T^{*} U$ if for any distribution $u$, there is an inclusion of $G^{s}$ wave front sets: $W F_{G^{s}}(u) \cap \Gamma \subset W F_{G^{s}}(L u) \cap \Gamma$.

The conjecture in question proposes a sufficient condition for the microlocal $G^{s}$ hypoellipticity of operators $L=\sum_{1 \leq j \leq k} X_{j}^{2}$, where the $X_{j}$ are real vector fields with real analytic coefficients in some open subset $V$ of $\mathbb{R}^{d}$, under the hypothesis that $\left\{X_{j}\right\}$ satisfies the bracket hypothesis of Hörmander [9]. Its formulation requires several definitions.

Denote by $\sigma_{j}$ the principal symbol of $X_{j}$, and by $T^{*} V$ the cotangent bundle of $V$ with the zero section deleted. Let $M \subset T^{*} V$ be a smooth submanifold. For the purposes of this paper, a submanifold $M^{\prime} \subset M$ of positive dimension will be said ${ }^{1}$ to be a bicharacteristic submanifold of $M$ if the tangent space of $M^{\prime}$ is orthogonal to the tangent space of $M$ with respect to the canonical symplectic form on $T^{*} V$, at every point of $M^{\prime}$.

Define $\mathcal{I}_{1}$ to be the ideal, in the ring of germs of real analytic functions on $T^{*} V$, generated by all the symbols $\sigma_{j}$. Inductively define $\mathcal{I}_{j+1}$ to be the ideal generated by $\mathcal{I}_{j}$ together with all Poisson brackets $\left\{f, \sigma_{i}\right\}$ such that $f \in \mathcal{I}_{j}$ and $1 \leq i \leq k$. Define $\Sigma_{j} \subset T^{*} V$ to be the zero variety of $\mathcal{I}_{j}$. Then $\mathcal{I}_{j} \subset \mathcal{I}_{j+1}$ and $\Sigma_{j} \supset \Sigma_{j+1}$ for all $j \geq 1$. The bracket hypothesis at a point $x \in V$ implies that $\Sigma_{m} \cap T_{x}^{*} V=\emptyset$ for some finite $m$. Under that hypothesis, define $m(x)$ to be the

Received June 6, 1997.

Research supported by NSF grant DMS 96-23007.

${ }^{1}$ A notion of bicharacteristic leaf is defined slightly differently by Treves [12]; it is not defined in [2]. Likewise the Poisson stratification introduced in [12] differs from that of [2]. These fine distinctions are not relevant to the simple examples treated in this article. 
smallest integer $m$ such that $\Sigma_{m} \cap T_{x}^{*} V=\emptyset$. A more refined invariant $m(x, \xi)$, defined at each point of $T^{*} V$, is the smallest integer such that $(x, \xi) \notin \Sigma_{m}$. Assuming for simplicity that each $\Sigma_{j}$ is a smooth manifold, define a second invariant, $\ell(x, \xi)$, to be the smallest index $j<m(x, \xi)$ such that for every conic neighborhood $\Gamma$ of $(x, \xi), \Sigma_{j} \cap \Gamma$ contains a bicharacteristic submanifold, provided such an index exists. Define $\ell(x, \xi)=m(x, \xi)$ if no such $j<m(x, \xi)$ exists.

Conjecture 1. (Bove and Tartakoff [2]) Let $L$ be a sum of squares of $C^{\omega}$ real vector fields, satisfying the bracket hypothesis at $x$. Suppose that there exists a neighborhood $V_{0}$ of $x$ such that each $\Sigma_{j} \cap T^{*} V_{0}$ is a smooth manifold. Then $L$ is microlocally $G^{s}$ hypoelliptic in a small conic neighborhood of $(x, \xi)$ for every $s \geq m(x, \xi) / \ell(x, \xi)$.

Modulo certain fine distinctions, this generalizes a conjecture of Treves [12] concerning the analytic case $s=1$.

In [6] we showed that the operators $\partial_{x_{1}}^{2}+x_{1}^{2 p} \partial_{x_{2}}^{2}+x_{1}^{2 q} \partial_{x_{3}}^{2}$ are $G^{s}$ hypoelliptic if and only if $s \geq \max (p / q, q / p)$, thereby demonstrating that the optimal exponent for Gevrey hypoellipticity is not always 1 or $m(x)$, but rather that a range of intermediate behavior arises. A refinement in terms of certain anisotropic generalizations of the Gevrey classes was then formulated and proved, by a different method, by Bove and Tartakoff [2]. Their conjecture is consistent with these examples.

In the present note basic examples of a different character will be analyzed. ${ }^{2}$ Their import is twofold: First, $G^{s}$ hypoellipticity may sometimes hold for a larger range of exponents than predicted by Conjecture 1. Second, the mechanism underlying the simpler examples of [6] is not the only factor influencing Gevrey hypoellipticity.

Consider

$$
L_{m, p}=\partial_{x}^{2}+\left(x^{m-1} \partial_{t}\right)^{2}+\left(t^{p} \partial_{t}\right)^{2}
$$

in $\mathbb{R}^{2}$. Assume that $m \geq 2$ and $p \geq 1$ are integers. Then any such $L$ is elliptic everywhere except where $(x, t)=0$; with coordinates $(x, t, \xi, \tau)$ for $T^{*} \mathbb{R}^{2}$, its characteristic variety is the line $\{x=t=\xi=0\} .{ }^{3}$

Theorem 2. $L_{m, p}$ is $G^{s}$ hypoelliptic for all s satisfying

$$
s^{-1} \leq 1-p^{-1}\left(1-m^{-1}\right)
$$

\footnotetext{
${ }^{2}$ More general results were announced in [4], based on the argument used below to derive Theorems 2 and 3. That argument works when a certain polynomial $\Theta$ arising in the theory of [4] is nonnegative on $\mathbb{R}^{2}$ and certain higher order terms are dominated by it, but a more elaborate argument for the general case contained a gap; it yields a strictly weaker conclusion than the desired Gevrey class hypoellipticity. The correctness of the most general statements in [4] is doubtful.

${ }^{3}$ Since the characteristic variety of $L_{m, p}$ consists of a discrete set of rays, $G^{s}$ hypoellipticity is equivalent to microlocal $G^{s}$ hypoellipticity for $L_{m, p}$.
} 
Modulo insignificant lower order terms, the operators (1.1) are generalizations of a fundamental example of Métivier [11]; their Poisson strata are discussed by Treves [12], Example 3.6. These operators fail to be analytic hypoelliptic, as follows from the method of [3] and [5].

In these examples $\Sigma_{j}=\{x=t=\xi=0\}$ for all $1 \leq j<m$, and $\Sigma_{m}=\emptyset$. Thus Conjecture 1 predicts $G^{s}$ hypoellipticity if and only if $s^{-1} \leq m^{-1}$. But $\ell(0,0,0, \tau)=1$ for all $\tau \neq 0$, and when $p \geq 2$, the reciprocal of the optimal exponent for $G^{s}$ hypoellipticity is $1-p^{-1}\left(1-m^{-1}\right)>1-\left(1-m^{-1}\right)=m^{-1}$.

The following variant of Theorem 2 can be proved by the same technique, and was also obtained by Bernardi, Bove and Tartakoff [1] and Matsuzawa [10]. Consider

$$
\mathcal{L}_{m, k, p}=\partial_{x}^{2}+\left(\left[x^{m-1}+x^{m-1-k} t^{p}\right] \partial_{t}\right)^{2} .
$$

Define $\tilde{p}=p(m-1) / k$.

Theorem 3. Suppose that $m-1, k, p$ are all even. Then $\mathcal{L}_{m, k, p}$ is $G^{s}$ hypoelliptic for all $s^{-1} \leq 1-\tilde{p}^{-1}\left(1-m^{-1}\right)$.

By an elaboration of the method of [3] and [5] we have shown the indicated range of $s$ to be optimal in Theorems 2 and 3, but the proofs are more involved than those of the positive results and will not be indicated here.

One interpretation of Theorem 2 is that not only the symplectic geometry of the varieties $\Sigma_{j}$, but also the ideals $\mathcal{I}_{j}$ themselves, influence Gevrey class hypoellipticity for $s<1$. We believe this also to be the case for $s=1$. The following examples may be of interest: let $L=X^{2}+Y^{2}$ in $\mathbb{R}^{3}$ with coordinates $(x, y, t)$ where $X=\partial_{x}$ and $Y=\partial_{y}+a(x, y) \partial_{t}, a \in C^{\omega}$ is real valued, and $\partial a / \partial x=$ $x^{2 p}+x^{2} y^{2}+y^{2 p}$ for some $p \geq 2$. Hypoellipticity of these operators depends only on $\partial a / \partial x$, rather than on $a$ itself. Conjecture 1 predicts analytic hypoellipticity for all $p \geq 2$. Indeed, $m=6$ for all $p$; the varieties $\Sigma_{j}$ are independent of $p$ for all $j \geq 2$, and they equal the symplectic manifold $\{(x, y, t ; \xi, \eta, \tau): x=\xi=$ $y=\eta=0\}$ for $2 \leq j<6$, and are empty for $j=6$. $L$ is known to be analytic hypoelliptic for $p=2[8]$, but existing methods of proof do not appear to be applicable for $p>2$. The ideals $\mathcal{I}_{j}$ have a somewhat different character when $p>2$ than when $p=2$.

After this paper was circulated we received preprints of Bernardi, Bove and Tartakoff [1] and of Matsuzawa [10] containing Theorems 2 and 3, with different methods of proof. The latter paper contains more general results as well.

\section{Proofs}

The method of proof of Theorem 2 is the same as that used in [5] and [6] to prove results in the positive direction. ${ }^{4}$ Fix $m, p$. For any linear partial

\footnotetext{
${ }^{4}$ This method does apply in somewhat greater generality, but our aim here is the analysis of the simplest relevant examples.
} 
differential operator $L$, denote by $L^{*}$ its adjoint. Write $y=(x, t), \eta=(\xi, \tau)$. The coordinate $t$ will sometimes be complex, whereas $x, \xi, \tau$ will remain real.

For any compactly supported distribution $u$ in $\mathbb{R}^{2}$, consider the FBI transform

$$
\mathcal{F} u(y, \eta)=\int u\left(y^{\prime}\right) \alpha\left(y-y^{\prime}\right) e^{i\left(y-y^{\prime}\right) \cdot \eta-\frac{1}{2}\langle\eta\rangle\left(y-y^{\prime}\right)^{2}} d y^{\prime},
$$

where $\left(y-y^{\prime}\right)^{2}$ is defined to be $\left(x-x^{\prime}\right)^{2}+\left(t-t^{\prime}\right)^{2},\langle\eta\rangle=\left(1+\eta^{2}\right)^{1 / 2}, \alpha(x, t)=$ $\left(1+\frac{i}{2} x \xi\langle\eta\rangle^{-1}\right)\left(1+\frac{i}{2} t \tau\langle\eta\rangle^{-1}\right)$, and the integral is interpreted in the sense of distributions if $u \notin L^{1}$. Then $u \in G^{s}$ in a neighborhood of some point $y_{0}$, if and only if there exist a neighborhood $V$ of $y_{0}$ and $\delta>0$ such that

$$
\mathcal{F} u(y, \eta)=O\left(\exp \left(-\delta\langle\eta\rangle^{1 / s}\right)\right)
$$

for all $(y, \eta) \in V \times \mathbb{R}^{2}$.

In proving $G^{s}$ hypoellipticity near $y_{0}$, we may assume $u$ to be supported in a small neighborhood of $y_{0}$, and $\mathcal{F}\left(L_{m, p} u\right)(y, \eta)$ to satisfy $(2.2)$ in $V \times \mathbb{R}^{d}$ for some smaller neighborhood $V$. Operators which are microlocally elliptic are microlocally $G^{s}$ hypoelliptic, so since the characteristic variety of $L_{m, p}$ is the line $x=t=\xi=0$, it suffices to prove (2.2) for $y$ near 0 and where $\eta=(\xi, \tau)$ with $|\tau| \geq|\xi|$ and $|\eta|$ large. Thus $|\tau| \sim|\eta|$.

Define

$$
\gamma(m, p)=1-p^{-1}\left(1-m^{-1}\right) .
$$

Then $0<\gamma(m, p)<1$, and we aim to prove $G^{s}$ hypoellipticity for all $s \geq$ $\gamma(m, p)^{-1}$.

The main step is the following lemma. Let $B_{\delta}=\left\{y \in \mathbb{C}^{2}:|y|<\delta\right\}$. Let $\tilde{y}=(\tilde{x}, \tilde{t}) \in \mathbb{R}^{2}$ be any point near 0 , and set

$$
E(x, t)=\exp \left(i(\tilde{t}-t) \tau-\frac{1}{2}\langle\eta\rangle^{\gamma}(\tilde{t}-t)^{2}\right)
$$

Lemma 2.1. Let $L=L_{m, p}$ and $\gamma=\gamma(m, p)$. Then for any sufficiently small constants $0<c_{1}<c_{2}<c_{3}$ there exists $\delta>0$ such that for each $\tilde{y} \in B_{c_{1}} \cap \mathbb{R}^{2}$ and each $\eta=(\xi, \tau) \in \mathbb{R}^{2}$ satisfying $|\xi| \leq|\tau|$, there exists $g \in C^{\infty}\left(B_{c_{3}} \cap \mathbb{R}^{2}\right)$ satisfying the following three conditions.

$$
\begin{aligned}
& L^{*}(g E)(y)= \\
& \alpha(\tilde{y}-y) e^{i(\tilde{x}-x) \xi-\frac{1}{2}\langle\eta\rangle(\tilde{x}-x)^{2}} E(y)+O\left(e^{-\delta\langle\eta\rangle^{\gamma}}\right) \quad \text { for } y \in B_{c_{3}} \cap \mathbb{R}^{2},
\end{aligned}
$$

$g$ extends to a holomorphic function of $t$ in $B_{c_{3}} \cap\left\{|\operatorname{Im}(t)|<\langle\eta\rangle^{\gamma-1}\right\}$ and

$$
g(y)=O(1) \text { in the } L^{2} \text { norm for } y \in B_{c_{3}} \cap\left\{|\operatorname{Im}(t)|<\langle\eta\rangle^{\gamma-1}\right\},
$$

and

$$
\begin{aligned}
& g(x, t)= \\
& O\left(e^{-\delta\langle\eta\rangle^{\gamma}}\right) \text { in the } L^{2} \text { norm for }(x, t) \in B_{c_{3}} \cap \mathbb{R}^{2} \text { where }|x|>c_{2} .
\end{aligned}
$$


A symbol " $O(\cdot)$ " connotes a bound uniform in $\eta, \tilde{y}, y$. Before discussing the proof, we indicate how the lemma leads to Theorem 2.

Lemma 2.2. Let $L$ be any linear partial differential operator satisfying the conclusion of Lemma 2.1 for some $\gamma \in(0,1]$. Then for any $s \geq \gamma^{-1}$ and for any sufficiently small neighborhood $U$ of 0 and any relatively compact $U^{\prime} \Subset U$, for any $u \in \mathcal{D}^{\prime}\left(\mathbb{R}^{2}\right)$ such that $L u \in G^{s}(U)$, there exists $\varepsilon>0$ such that $\mathcal{F} u(y, \eta)=O\left(\exp \left(-\varepsilon|\eta|^{1 / s}\right)\right)$ as $|\eta| \rightarrow \infty$, uniformly for $y \in U^{\prime}$, provided that $\eta=(\xi, \tau)$ where $|\tau| \geq|\xi|$.

Sketch of proof. The easy proof is essentially identical to the argument immediately following the statement of Lemma 3.1 of [6], so we merely recall its outline. Suppose that $L u \in G^{s}\left(U^{\prime}\right)$, where $s=\gamma(m, p)^{-1}$.

Begin by rewriting the integral defining $\mathcal{F} u$ by substituting

$$
\alpha\left(y-y^{\prime}\right) \exp \left(i\left(y-y^{\prime}\right) \cdot \eta-\frac{1}{2}\langle\eta\rangle\left(y-y^{\prime}\right)^{2}\right)=L^{*}(g E)+O\left(\exp \left(-\delta\langle\eta\rangle^{\gamma}\right)\right)
$$

The second term leads to an error of the desired order of magnitude. Integrating by parts leads to a main term $\int g E \cdot L u$; boundary terms are negligible because $\exp \left(-\frac{1}{2}\langle\eta\rangle\left(y-y^{\prime}\right)^{2}\right)$ is $O(\exp (-c\langle\eta\rangle))$ away from the diagonal.

Next, because $L u \in G^{s}$, conclusion (5) of Theorem 2.3 of [6] asserts that it is possible to decompose $L u$ as $v+R$ where $v$ is holomorphic with respect to $t$ and is $O(1)$ in the region $|\operatorname{Im}(t)|<\langle\eta\rangle^{\gamma-1}$, and $R$ is $O\left(\exp \left(-\varepsilon\langle\eta\rangle^{\gamma}\right)\right)$ in the real domain. $R$ again leads to an acceptable error. Finally the contribution of $v$ is treated by shifting the contour of integration with respect to $t$ into the complex domain so as to pick up a factor of $\exp \left(-c\langle\eta\rangle^{\gamma}\right)$ from the factor $\exp (i(\tilde{t}-t) \tau)$ in $E$.

Any linear differential operator with analytic coefficients is microlocally $G^{s}$ hypoelliptic for all $s \geq 1$ in any conic open set where its principal symbol does not vanish. Therefore for any operator $L$ that is elliptic where $|\xi| \geq|\tau|$, under the hypotheses of the preceding lemma, one has also a decay estimate $O\left(\exp \left(-\varepsilon|\eta|^{1 / s}\right)\right)$ wherever $L$ is elliptic. In particular, the operators of Theorem 2 are elliptic where $|\xi| \geq|\tau|$.

To prove Theorem 2, we couple these decay estimates with the FBI transform characterization (2.2) of $G^{s}$, to conclude that any $L$ that satisfies the conclusion of Lemma 2.1, and is elliptic where $|\xi| \geq|\tau|$, is $G^{s}$ hypoelliptic in a neighborhood of the origin for all $s \geq \gamma^{-1}$. In particular, $L_{m, p}$ is $G^{s}$ hypoelliptic for all $s \geq \gamma(m, p)^{-1}$. Thus Theorem 2 is proved, modulo the proof of Lemma 2.1.

We now discuss the proof of Lemma 2.1. Fix $(\tilde{x}, \tilde{t})$ and $\eta=(\xi, \tau)$ where $|\tau| \geq|\xi|$. One has

$$
\begin{aligned}
& E^{-1} L^{*} E= \\
& \partial_{x}^{2}+\left(x^{m-1}\left[\partial_{t}-i \tau+\langle\eta\rangle(\tilde{t}-t)\right]\right)^{2}+\left(\left[\partial_{t}-i \tau+\langle\eta\rangle(\tilde{t}-t)\right] t^{p}\right)^{2} .
\end{aligned}
$$


Write

$$
E^{-1} L^{*} E=\mathcal{A}+\mathcal{R} \quad \text { where } \quad \mathcal{A}=\partial_{x}^{2}-\tau^{2} x^{2(m-1)}-\tau^{2} t^{2 p} .
$$

$\mathcal{A}$ acts on functions of $(x, t)$; we also write $A_{t}=\partial_{x}^{2}-\tau^{2} x^{2(m-1)}-\tau^{2} t^{2 p}$ to denote the same operator, acting on functions of $x$ alone and depending on a parameter $t$.

The construction of the approximate solution $g$ sought in Lemma 2.1 transpires in various Sobolev type spaces. Henceforth let $\gamma=\gamma(m, p)$. Define

$$
w_{\tau}(x, t)=\left(\tau^{2 / m}+\tau^{2} x^{2(m-1)}+\tau^{2}|t|^{2 p}\right)^{1 / 2},
$$

for $(x, t) \in \mathbb{R} \times \mathbb{C}$. Fix a nonnegative auxiliary function $v \in C^{\infty}(\mathbb{R})$ such that $v \equiv 0$ in a neighborhood of $\left\{|x| \leq c_{1}\right\}$, and $v \equiv 1$ in a neighborhood of $\left\{|x| \geq c_{2}\right\}$. For any open set $\Omega \subset \mathbb{C}^{1}$, for $k \in\{0,1,2\}$, define $\mathcal{H}_{\tau}^{k}(\mathbb{R} \times \Omega)$ to consist of all measurable functions $f(x, t)$ defined on $\mathbb{R} \times \Omega$ that are holomorphic in $t$ for almost every $x$, and for which the following norms are finite:

$$
\begin{aligned}
\|f\|_{\mathcal{H}_{\tau}^{0}(\mathbb{R} \times \Omega)}^{2} & =\iint_{\mathbb{R} \times \Omega}|f(x, t)|^{2} w_{\tau}(x, t)^{-2} e^{\rho|\tau| v(x)} d x d t d \bar{t} \\
\|f\|_{\mathcal{H}_{\tau}^{1}(\mathbb{R} \times \Omega)}^{2} & =\iint_{\mathbb{R} \times \Omega}\left(\left|\partial_{x} f(x, t)\right|^{2} w_{\tau}(x, t)^{-2}+|f(x, t)|^{2}\right) e^{\rho|\tau| v(x)} d x d t d \bar{t} \\
\|f\|_{\mathcal{H}_{\tau}^{2}(\mathbb{R} \times \Omega)}^{2} & =\iint_{\mathbb{R} \times \Omega}\left(\left|\partial_{x}^{2} f(x, t)\right|^{2} w_{\tau}(x, t)^{-2}+\left|\partial_{x} f(x, t)\right|^{2}\right. \\
& \left.+|f(x, t)|^{2} w_{\tau}(x, t)^{2}\right) e^{\rho|\tau| v(x)} d x d t d \bar{t} .
\end{aligned}
$$

These spaces and norms depend on the parameter $\rho$, which may for the present be any real number but will ultimately be chosen to be small but strictly positive. There are corresponding spaces of functions defined on $\mathbb{R}$, depending on a parameter $t \in \mathbb{C}$ :

$$
\begin{aligned}
\|f\|_{\mathcal{H}_{\tau, t}^{0}(\mathbb{R})}^{2} & =\int_{\mathbb{R}}|f(x)|^{2} w_{\tau}(x, t)^{-2} e^{\rho|\tau| v(x)} d x \\
\|f\|_{\mathcal{H}_{\tau, t}^{1}(\mathbb{R})}^{2} & =\int_{\mathbb{R}}\left(\left|\partial_{x} f(x)\right|^{2} w_{\tau}(x, t)^{-2}+|f(x)|^{2}\right) e^{\rho|\tau| v(x)} d x \\
\|f\|_{\mathcal{H}_{\tau, t}^{2}(\mathbb{R})}^{2} & =\int_{\mathbb{R}}\left(\left|\partial_{x}^{2} f(x)\right|^{2} w_{\tau}(x, t)^{-2}+\left|\partial_{x} f(x)\right|^{2}+|f(x)|^{2} w_{\tau}(x, t)^{2}\right) e^{\rho|\tau| v(x)} d x
\end{aligned}
$$

The definitions ensure that $\mathcal{A}$ maps $\mathcal{H}_{\tau}^{2}(\mathbb{R} \times \Omega)$ boundedly to $\mathcal{H}_{\tau}^{0}(\mathbb{R} \times \Omega)$, uniformly in $\Omega, \tau$, under the standing hypotheses that $|\tau| \geq|\xi|$ and $|\tau| \geq 1$. Likewise $A_{t}$ maps $\mathcal{H}_{\tau, t}^{2}(\mathbb{R})$ boundedly to $\mathcal{H}_{\tau, t}^{0}(\mathbb{R})$, uniformly in $\tau \in \mathbb{R}, t \in \mathbb{C}$. 
Lemma 2.3. There exists $c_{0}>0$ such that for all sufficiently small $|\rho|$ and all $\tau \neq 0, A_{t}: \mathcal{H}_{\tau, t}^{2}(\mathbb{R}) \mapsto \mathcal{H}_{\tau, t}^{0}(\mathbb{R})$ is invertible, uniformly in $t \in \mathbb{C}, \tau \in \mathbb{R}$ provided that

$$
|\operatorname{Im}(t)| \leq c_{0}|\tau|^{\gamma(m, p)-1}
$$

Proof. The proof is based on the inequality

$$
-\operatorname{Re}\left\langle A_{t} f, f\right\rangle \geq c \int_{\mathbb{R}}\left(\left|\partial_{x} f\right|^{2}+w(x, t)^{2}|f|^{2}\right) d x \quad \text { for all } f \in C_{0}^{2}(\mathbb{R}),
$$

where $\langle f, g\rangle=\int_{\mathbb{R}} f \bar{g} d x$. To prove this write

$$
-\operatorname{Re}\left\langle A_{t} f, f\right\rangle=\left\|\partial_{x} f\right\|^{2}+\int_{\mathbb{R}} x^{2(m-1)} \tau^{2}|f|^{2} d x+\int \tau^{2} \operatorname{Re}\left(t^{2 p}\right)|f|^{2} d x .
$$

One has

$$
\left\|\partial_{x} f\right\|^{2}+\int x^{2(m-1)} \tau^{2}|f|^{2} d x \geq c \tau^{2 / m} \int|f|^{2} d x
$$

as follows from the case $\tau=1$ by scaling. Moreover

$$
\operatorname{Re}\left(t^{2 p}\right) \geq c(\operatorname{Re}(t))^{2 p}-C(\operatorname{Im}(t))^{2 p}
$$

for some $c, C \in \mathbb{R}^{+}$. The hypothesis $(2.10)$ restricting the imaginary part of $t$ implies

$$
\tau^{2}(\operatorname{Im}(t))^{2 p} \leq c_{0}^{2 p} \tau^{2+2 p(\gamma-1)} .
$$

The exponent is $2+2 p(\gamma-1)=2-2 p\left(p^{-1}\left(1-m^{-1}\right)\right)=2 m^{-1}$. Combining all these ingredients yields (2.11), provided that $c_{0}$ is chosen to be sufficiently small.

The conclusion of the lemma follows easily from (2.11) as in [3], Lemma 3.1 and [6], Lemma 3.3, because

$$
e^{\rho|\tau| v / 2} A_{t} e^{-\rho|\tau| v / 2}-A_{t}=O(|\rho|)
$$

as an operator from $\mathcal{H}_{\tau, t}^{2}(\mathbb{R})$ to $\mathcal{H}_{\tau, t}^{0}(\mathbb{R})$; this holds because $v \equiv 0$ in a neighborhood of the origin while the term $|\tau| x^{m-1}$ in the definition of $w$ is strictly positive on the support of $v$. For further details see the proof of Lemma 3.3 of $[6]$.

Corollary 2.4. If $c_{0}$ is chosen to be sufficiently small then for any open set $\Omega \subset \mathbb{C}^{1}$ contained in the region where $|\operatorname{Im}(t)|<c_{0}|\tau|^{\gamma(m, p)-1}$, the operator $\mathcal{A}: \mathcal{H}_{\tau}^{2}(\mathbb{R} \times \Omega) \mapsto \mathcal{H}_{\tau}^{0}(\mathbb{R} \times \Omega)$ is invertible, uniformly in $\tau, \Omega$. 
Let

$$
\begin{aligned}
\Omega_{1} & =\left\{t \in \mathbb{C}:|\operatorname{Re}(t)|<2 \text { and }|\operatorname{Im}(t)|<\frac{c_{0}}{2}|\tau|^{\gamma-1}\right\}, \\
\Omega_{\infty} & =\left\{t \in \mathbb{C}:|\operatorname{Re}(t)|<1 \text { and }|\operatorname{Im}(t)|<\frac{c_{0}}{4}|\tau|^{\gamma-1}\right\},
\end{aligned}
$$

Let $\Lambda \in \mathbb{R}^{+}$be a large constant to be chosen below. Given a large $\tau$, choose an integer $N$ so that $\left.\left|N-\Lambda^{-1}\right| \tau\right|^{\gamma} \mid<1$. For $2 \leq j \leq 2 N$ construct open sets $\Omega_{j} \subset \mathbb{C}$, depending on $\tau$, with $\Omega_{\infty}=\Omega_{2 N} \Subset \Omega_{2 N-1} \Subset \cdots \Subset \Omega_{1}$ satisfying

$$
\text { distance }\left(\Omega_{j+1}, \partial \Omega_{j}\right) \geq c \Lambda|\tau|^{-1} \text {. }
$$

Here $c$ is a small constant, independent of $\tau, \Lambda, j$.

Lemma 2.5. $\mathcal{R}: \mathcal{H}_{\tau}^{2}\left(\mathbb{R} \times \Omega_{j}\right) \mapsto \mathcal{H}_{\tau}^{0}\left(\mathbb{R} \times \Omega_{j+2}\right)$ is bounded, with norm $O\left(\Lambda^{-1}+\right.$ $\left.c_{1}+c_{0}\right)$, uniformly in $\tau$.

Proof. By Cauchy's inequality relating the derivative of a holomorphic function to its $L^{1}$ norm over a ball, $\partial_{t}$ maps each space $\mathcal{H}_{\tau}^{k}\left(\mathbb{R} \times \Omega_{j}\right)$ boundedly to $\mathcal{H}_{\tau}^{k}\left(\mathbb{R} \times \Omega_{j+1}\right)$, with norm $O\left(\right.$ distance $\left.\left(\Omega_{j+1}, \partial \Omega_{j}\right)^{-1}\right)=O\left(\Lambda^{-1}|\tau|\right)$. The norms are defined so that the multiplication operators $\tau t^{p}$ and $\tau x^{m-1}$ map $\mathcal{H}_{\tau}^{k}\left(\mathbb{R} \times \Omega_{j}\right)$ to $\mathcal{H}_{\tau}^{k-1}\left(\mathbb{R} \times \Omega_{j}\right)$ with uniformly bounded norms. Furthermore, the extra factors of $\tilde{t}-t$ in the definition (2.8),(2.9) for $\mathcal{R}$ contribute an additional factor to these bounds which is $O\left(c_{1}+c_{0}\right)$. Combining these estimates yields the lemma. For further details see the proofs of Lemma 3.4 of [6], and of the first display at the top of page 319 of [3].

Set $\psi(y)=\alpha(\tilde{y}-y) e^{i(\tilde{x}-x) \xi-\frac{1}{2}\langle\eta\rangle(\tilde{x}-x)^{2}}$. To attempt to solve $(\mathcal{A}+\mathcal{R}) g \approx \psi$ we define

$$
g=\sum_{j=0}^{N}(-1)^{j}\left(\mathcal{A}^{-1} h \mathcal{R}\right)^{j} \mathcal{A}^{-1} \psi,
$$

where $h \in C_{0}^{\infty}(\mathbb{R})$ is $\equiv 1$ where $|x| \leq c_{3}$. Thus

$$
(\mathcal{A}+h \mathcal{R}) g=\psi \pm \mathcal{E}
$$

where

$$
\mathcal{E}=\left(h \mathcal{R} \mathcal{A}^{-1}\right)^{N+1} \psi
$$

Note that $\psi \in \mathcal{H}_{\tau}^{0}\left(\mathbb{R} \times \Omega_{1}\right)$ with norm $O(1)$, provided $\rho>0$ is chosen to be sufficiently small. If $\Lambda$ is chosen to be sufficiently large and $c_{0}, c_{3}$ to be sufficiently small then applying Lemmas 2.3 and 2.5 in turn $N$ times yields

$$
\mathcal{E}=O(\exp (-\varepsilon N))=O\left(\exp \left(-\varepsilon^{\prime}|\tau|^{\gamma}\right)\right)
$$

for some $\varepsilon, \varepsilon^{\prime}>0$, in the $\mathcal{H}_{\tau}^{0}\left(\mathbb{R} \times \Omega_{\infty}\right)$ norm.

Because $v(x)>0$ for $|x| \geq c_{2}$ and $\rho>0$, the weight $e^{\rho|\tau| v(x)}$ in the definitions of the $\mathcal{H}_{\tau}^{k}$ norms ensures that $g=O\left(\exp \left(-\varepsilon|\tau|^{\gamma}\right)\right)$ in the $L^{2}(d x d t)$ norm for 
such $x$. In the region $|x|<c_{3}$ of interest, the auxiliary function $h$ is $\equiv 1$, hence $(\mathcal{A}+\mathcal{R}) g \equiv \psi+\mathcal{E}$. This approximate solution $g$ thus has all properties required of it in Lemma 2.1 .

The main change needed to obtain Theorem 3 is to modify the weight $w(x, t)$ used in the definitions of the $\mathcal{H}_{\tau}^{k}$ and $\mathcal{H}_{\tau, t}^{k}$ norms to

$$
\left(\tau^{2 / m}+\tau^{2 /(m-1-k)}|t|^{2 p}+\tau^{2}\left[x^{2(m-1)}+x^{2(m-1-k)}|t|^{2 p}\right]\right)^{1 / 2} .
$$

Remark. The limiting effect preventing this analysis from establishing $G^{s}$ hypoellipticity for a larger range of exponents $s$ is the failure of $A_{t}$ to be invertible for $t$ outside of a complex region which shrinks to the real axis as $|\tau| \rightarrow \infty$; the rate of shrinkage dictates the optimal Gevrey class $G^{s}$. This phenomenon is the essence of [5] and [3].

For the operators studied in [6] and [2], the limitation on $s$ comes about in a different way. Application of the FBI transform $\mathcal{F}$ as above leads to unacceptable error terms, so variants $\mathcal{F}_{\gamma}$ adapted to specific Gevrey classes were employed instead in [6] in order to obtain smaller error terms.

\section{References}

1. E. Bernardi, A. Bove, and D. Tartakoff, On a conjecture of F. Treves: Analytic hypoellipticity and Poisson strata, preprint.

2. A. Bove and D. Tartakoff, Optimal non-isotropic Gevrey exponents for sums of squares of vector fields, preprint.

3. M. Christ, The Szegö projection need not preserve global analyticity, Ann. of Math. 143 (1996), 301-330.

4. _ On local and global analytic and Gevrey hypoellipticity, Journees "Equations aux derivees partielles", Saint-Jean-de-Monts, Mai-Juin 1995.

5. __ Analytic hypoellipticity in dimension two, MSRI preprint 1996-009, submitted.

6. __ Intermediate optimal Gevrey exponents occur, Comm. Partial Diff. Eq. 22 (1997), 359-379.

7. Hypoellipticity in the infinitely degenerate regime, preprint.

8. A. Grigis and J. Sjöstrand, Front d'onde analytique et sommes de carrés de champs de vecteurs, Duke Math. J. 52 (1985), 35-51.

9. L. Hörmander, Hypoelliptic second order differential equations, Acta Math. 119 (1967), 147-171.

10. T. Matsuzawa, Gevrey hypoellipticity for Grushin operators, Pub. RIMS Kyoto University, to appear.

11. G. Métivier, Non-hypoellipticité analytique pour $D_{x}^{2}+\left(x^{2}+y^{2}\right) D_{y}^{2}$, C. R. Acad. Sci. Paris 292 (1981), 401-404.

12. F. Treves, Symplectic geometry and analytic hypo-ellipticity, preprint.

Department of Mathematics, University of California, Berkeley, CA 94720

E-mail address: mchrist@math.berkeley.edu 